\title{
Light therapy and the management of winter depression
}

\author{
John M. Eagles
}

\begin{abstract}
Much the most common type of seasonal affective disorder at temperate latitudes is recurrent winter depression, which probably affects around $3 \%$ of adults in the UK to a clinically significant degree. In this article, diagnosis and presentation are discussed and symptoms are contrasted with those of nonseasonal depression. Aetiology and epidemiology, with regard to age, gender and latitude of residence, are described. Sufferers are often treated with light therapy, and this is described in some detail, with mention of effectiveness, prediction of outcome, timescales of response, side-effects, use of lightboxes and alternatives to lightboxes. Other general aspects of the management of seasonal affective disorder, including the use of antidepressant medication, are also outlined.
\end{abstract}

The main purpose of this article is to discuss the use of light therapy in seasonal affective disorder. There are two recognised forms of this disorder. Recurrent summer depressions occur in people living close to the equator, but are very rare in those who live in temperate climates. This article focuses solely on recurrent winter depression, which is common at temperate latitudes, and for which light therapy is widely used. In order to make sense of light therapy for winter depression, my discussion of this topic is preceded by a description of seasonal affective disorder and of other approaches to its management.

\section{Historical perspective on seasonal affective disorder}

The notion of seasonal fluctuations in well-being is not a new one. In $400 \mathrm{BC}$, Hippocrates wrote that 'it is chiefly the changes of seasons which produce diseases', although he considered changes in temperature, rather than in light levels, to be the crucial aetiological factor. By 200 AD, Aretaeus was advocating light therapy, writing that 'lethargics are to be laid in the light and exposed to the rays of the sun (for the disease is gloom)'. It was perhaps Wilhelm Griesinger, writing in 1855, who produced the first clear account of seasonal affective disorder, describing 'cases where regularly at one particular season - for example in winter - a profound melancholia has supervened, which in spring passes into mania, which again in autumn gradually gives way to melancholia'. In the modern era, it was in 1984 that Rosenthal et al published their seminal paper describing a group of patients with seasonal affective disorder and the beneficial effects of bright artificial light on patients' symptoms. This 'rediscovery' of the disorder spawned renewed interest in its identification and management.

\section{Symptoms of winter depression}

Seasonal affective disorder is not recognised as a specific disorder in ICD-10, but it is in DSM-IV, as recurrent depressive episodes with a seasonal pattern (American Psychiatric Association, 1994). It is this seasonal recurrence, perhaps self-evidently, that is the hallmark of winter depression. Symptoms typically commence in autumn or winter, peak between December and February and remit during spring and summer, during which seasons up to a third of patients become hypomanic, usually to a mild degree.

Many of the symptoms of winter depression are also characteristic of non-seasonal depressive episodes: low mood (albeit usually without prominent diurnal variation), loss of interest, anhedonia, anergia, poor motivation, low libido, anxiety, irritability and social withdrawal. Other

John Eagles is a consultant psychiatrist at Royal Cornhill Hospital (Cornhill Road, Aberdeen AB25 2ZH, UK) and an honorary reader in mental health at the University of Aberdeen. He has been assessing and treating patients with seasonal affective disorder for 10 years. His other research interests include eating disorders and suicide. 
symptoms occur that are much more characteristic of winter depression. About three-quarters of patients experience a significant increase in duration of sleep, while perceiving their sleep to be of poor quality. This is associated with daytime somnolence, which often peaks in the late afternoon. A similar proportion of patients experience an increase in weight and in appetite, during which cravings for carbohydrate and chocolate are frequently prominent. As described below, several of these symptoms often interact, giving rise to further deteriorations in well-being.

Onset is typically between 20 and 30 years of age, although symptoms may often be recalled much earlier in life. Winter depression does occur in children; fatigue, irritability and academic problems at school are often the presenting features.

\section{Differential diagnosis and comorbidity}

In psychiatric practice, the most common differential diagnosis (based on symptoms and seasonality of recurrences) will be that of recurrent non-seasonal depressive disorder. Perhaps the most important reason for distinguishing the two conditions relates to the instigation of light therapy. Many patients will experience winter fatigue and somnolence as greater problems than depression itself, contributing to a greater likelihood that patients and doctors might somatise the disorder. In our study of winter depression in primary care (Eagles et al, 2002), in comparison with control subjects, patients underwent many more investigations (notably for suspected anaemia or hypothyroidism) and received more prescriptions covering a wide range of different physical ailments. Winter depression had been diagnosed only very rarely by the patients' general practitioners.

As with non-seasonal depression, several conditions are not uncommonly comorbid with seasonal affective disorder. Anxiety symptoms are common concomitants of winter depression (Thompson \& Isaacs, 1988) and, although estimates have varied, it seems likely that 5-20\% of patients experience panic disorder. Patients with eating disorders, most notably bulimia nervosa, frequently experience a winter exacerbation of their symptoms (Blouin et al, 1992). Women with pre-menstrual depression report increased seasonal fluctuations in well-being, as do patients with chronic fatigue syndrome. Several studies have found high rates of comorbid personality disorders in seasonal affective disorder, although the prevalence of these diagnoses tends to reduce once patients become euthymic (ReichbornKjennerud et al, 1997).

\section{Does seasonal affective disorder exist?}

Scepticism about the existence of seasonal affective disorder is encountered among psychiatrists, some of whom feel that the distinction from other types of recurrent depression is irrelevant. Other psychiatrists go further, asserting that it is a potentially misleading and harmful myth for people who seek illegitimate refuge in the sick role. The high demands that patients with the disorder make on several areas of the health service, as mentioned above (Eagles et al, 2002), can be seen as support for this view. Furthermore, longitudinal studies reveal that the course of patients diagnosed with seasonal affective disorder can be quite variable; some remit, some pursue a continuing seasonal pattern and some develop a non-seasonal pattern of recurrence. While a degree of caution is no doubt justified, a diagnosis is ultimately useful if it helps with management. Although a watertight case can not be made, I would suggest that the evidence for the efficacy of light therapy is sufficiently strong to justify the continuing diagnosis of winter depression.

\section{Epidemiology of winter depression}

Most people who live at temperate latitudes will, to a certain extent, recognise some of the symptoms of winter depression in themselves. These symptoms occur across a spectrum of severity, and there is contention as to where an appropriate line might be drawn between 'normality' and 'disorder'.

Kasper et al (1989) introduced the term 'subsyndromal seasonal affective disorder' (otherwise known as 'winter blues') to describe a milder form of the condition; their group felt this to be a useful diagnosis, since symptoms also responded to light therapy in a small trial. Inclusion of this group, however, served to further 'lower the bar' with regard to the prevalence of treatable winter depression, giving rise to usual population rates in the Seasonal Pattern Assessment Questionnaire (SPAQ; Rosenthal et al, 1987), the most widely used screening instrument for seasonal affective disorder, in excess of $20 \%$. That the SPAQ yields an overinclusive estimate of the prevalence of the disorder has since been demonstrated, but much of what we know about the epidemiology of seasonal affective disorder derives from SPAQ screening studies and claims of such high prevalence did little in some quarters to render the diagnosis credible. More recent studies in the UK, utilising interviews and DSM-IV criteria after screened patients had fulfilled SPAQ criteria for 


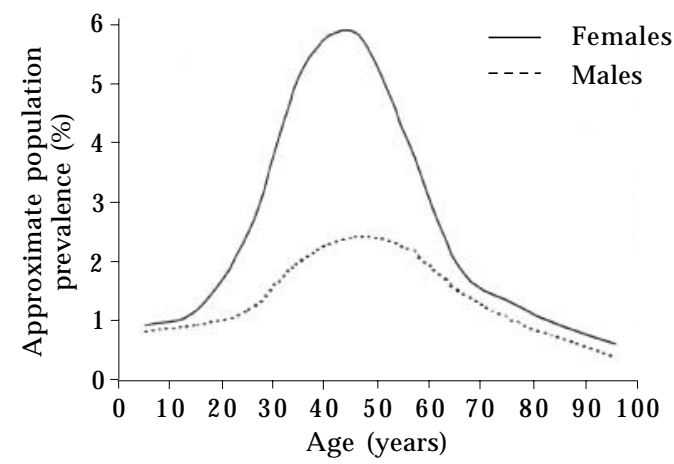

Fig. 1 Prevalence of winter depression by age and gender.

winter depression, gave rise to estimated adult community prevalence rates of $3.5 \%$ in Aberdeen (Eagles et al, 1999) and $2.4 \%$ in North Wales (Michalak et al, 2001).

In their large study of seasonal affective disorder among North American children, Swedo et al (1995) found that the low prevalence in young children of both genders increased markedly at puberty in girls but not in boys. Rates of the disorder reach a peak among women in their reproductive years (Fig. 1), with a decline in old age, so that the genders are again fairly evenly affected.

Early North American studies showed a clear relationship of increasing prevalence with increasing latitude of residence. More recently, Mersch et al (1999) concluded that this relationship does not pertain in European studies. It is possible that this disparity relates to geographical mobility within populations. People who are born and continue to reside at quite northern latitudes, for example, the Icelandic population studied by Magnusson \& Stefansson (1993), experience relatively low rates of seasonal affective disorder. It is likely that among such populations, winter depression would confer both a reproductive disadvantage and an increased likelihood of moving south. Conversely, moving to live further away from the equator probably gives rise to an increased risk of winter depression. Magnusson (2000) has published a useful overview of epidemiological studies of seasonal affective disorder.

\section{Aetiological considerations}

In straightforward terms, light deprivation can be seen as the main cause of winter depression. Seasonal changes in daylight hours exert profound effects on the biology of the animal kingdom, notably with regard to breeding patterns and hibernation, to which winter depression has been likened. The increased likelihood during the past century of working indoors has been linked to the increased recognition of seasonal affective disorder and patients' symptoms in winter improve in brighter weather.

Allied to this is the 'phase-shift hypothesis' (as occurs in jetlag) described by Lewy et al (1987). The circadian rhythms of people with winter depression are seen as phase delayed with late onset of melatonin secretion, a pattern that can be corrected with morning light.

At the neurotransmitter level, serotonin has the clearest links with seasonal affective disorder. Various parameters of serotonergic function fluctuate seasonally, serotonin is linked intimately with regulation of appetite and sleep, and tryptophan depletion gives rise to worsening of symptoms in recovered patients. Evidence for the likely importance of noradrenergic mechanisms has also emerged (Neumeister et al, 1998).

Evolutionary factors might play an aetiological role. At one stage, it may have been advantageous to have been energetic and to require little sleep in the summer and to be anergic and sleepy in winter. This may have applied especially to women and their offspring, since it would be optimal to become pregnant in summer, resulting in childbirth in spring, when food is more plentiful and the weather is becoming warmer.

Eleven chapters in a book edited by Partonen \& Magnusson (2001) are devoted to discussion of the pathogenesis of seasonal affective disorder.

\section{General aspects of the management of winter depression}

In my experience, management of winter depression is aided by an appreciation of the interaction of different symptom areas and of the fact that different approaches are usually merited for different levels of severity.

\section{Symptom interactions}

Lethargy, somnolence and low mood are usually the first symptoms to emerge in the autumn. As carbohydrate craving commences, those affected usually reduce their levels of exercise; many people with winter depression exercise regularly in the summer and stop completely in the winter. Both of these factors contribute to weight gain, which in turn can contribute to irritability, deteriorating interpersonal relationships and social withdrawal. 


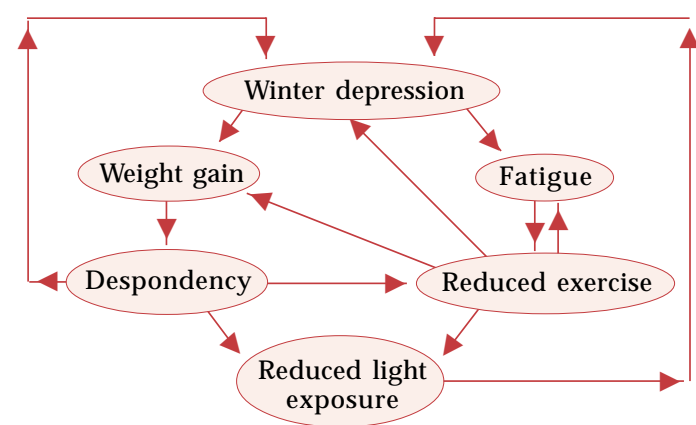

Fig. 2 Interacting factors in winter depression.

Figure 2 summarises some of these 'SAD cycles' in winter depression. Perhaps obviously, interventions that are helpful for any symptom area can help to reverse a downward spiral. For example, encouraging patients to continue with exercise throughout autumn and winter can improve mood, reduce irritability and stress, lessen the likelihood of weight gain and promote social contact. An association between seasonal fluctuations in well-being and negative life events and low levels of social support has recently been described (Michalak et al, 2003). The causal direction of this relationship was not clear, and these factors might also promote a downward spiral. Depression and social withdrawal give rise to a reduction in exposure to natural daylight, and people with winter depression experience less light exposure than the general population in winter; again, cause and effect can be debated, but increased natural light exposure is to be encouraged.

\section{Some principles of management}

While there are different strategies for the treatment of winter depression that hinge largely on severity, there are several aspects of management that can be generalised to most patients, and here I give a synopsis of my approach. It is usually helpful if the interrelationship of symptom areas is discussed and recognised by patients, and that they appreciate that improvement in one area is likely to give rise to 'knock-on' improvement in others. It is often relevant to discuss healthy eating strategies. In general, sufferers should attempt to get into regular exercise routines in the summer and to persevere with these to the best of their ability during the winter months. As much of the exercise as possible should be outdoors during daylight. If they can be afforded, sunny winter holidays (January may be the ideal month for many) are physiologically and psychologically beneficial. Social contact should be continued if possible, and in general it is helpful if friends and relatives know to anticipate, and attempt to tolerate, some degree of lethargy, low mood and irritability.

To my knowledge, the efficacy of psychological therapies in winter depression has not been formally evaluated. It seems unlikely, however, that the place of psychological approaches in winter depression is all that different from their place in other recurrent depressive disorders, and many of the general aspects of management already described are designed partly to promote active mastery over, as opposed to passive submission to, recurrent winter symptoms. It is interesting that Rohan et al (2003) have recently reported that trial participants with winter depression tended to have more negative automatic thoughts than controls throughout the year, and that a ruminative thinking style in autumn correlated with severity of winter symptoms. This serves to support the use of a broadly active mastery or a cognitive-behavioural model of management. Reformulating negative thoughts is often helpful. For example, sufferers tend to focus gloomily on the relative certainty with which depressions recur, rather than on the knowledge that they will remit. Most patients can be reassured that they will learn more about the management of their condition each winter, and thus manage it better during the next. It is usually helpful to regard the shortest day as something of a 'winning post' and to know that by Christmas each day is becoming a few minutes longer.

\section{Mild winter depression}

It would probably be justifiable to classify more than half of the UK population as experiencing mild symptoms of winter depression, notably a decrease in energy, enhanced appetite and increased duration of sleep. The general principles of management discussed above suffice for what should be seen as normal seasonal fluctuation in physiological functioning at temperate latitudes.

\section{Moderate to severe winter depression}

When symptoms are sufficiently severe to interfere markedly with the ability to function socially and/ or at work then, in addition to an active mastery strategy, physical treatments are indicated in the form of light therapy and/or antidepressants. 


\section{Light therapy or antidepressants?}

As a general rule, the more that the typical winter symptoms (such as hypersomnia, carbohydrate craving and weight gain) predominate, the more likely that light therapy should be the treatment of first choice. If, however, winter episodes are characterised by early morning wakening and weight loss, and especially when patients describe non-seasonal recurrences, antidepressants should be advised. Patients usually have a preference, and in my experience this is usually for 'natural' light therapy over 'chemical' antidepressants. However, as most services do not provide lightboxes on loan, the majority of patients have to acquire their own. Clearly, this is more expensive and troublesome to the patient, and results in more delay, than the prescription of antidepressants. For busy people, whose activities are already constrained by winter symptoms, taking antidepressants may seem a more effective use of time than light therapy. Studies have not been conducted to allow an evidence-based choice to be made between light therapy and antidepressants. In more severe winter depression, the two treatments are often used together.

\section{Antidepressant treatment}

There is also a woeful lack of evidence on which to base antidepressant management of winter depression. Given the usual somnolent, anergic symptom profile, sedative antidepressants are usually avoided and selective serotonin reuptake inhibitors (SSRIs) (for which there is a little evidence of efficacy in this population) are the usual first choice. For most patients, SSRIs are non-sedative and, especially when taken over a period of less than 6 months, tend not to exacerbate potential weight gain. There is no logical reason to select one SSRI over another, although the small amount of evidence is for the efficacy of sertraline and fluoxetine (for a review see Thompson, 2001). If SSRIs prove unsuccessful, venlafaxine is a logical alternative, and it sometimes proves more energising. Reboxetine can have a similar effect. Less sedative tricyclics such as imipramine can be used. Counterintuitively, given its sedative properties, an open trial of mirtazapine found it to be efficacious and well tolerated (Hesselmann et al, 1999).

In patients with established winter recurrences, it is usual to instigate treatment as soon as symptoms re-emerge in the autumn and to phase out treatment in spring. For patients who also experience nonseasonal episodes, year-round prophylaxis may be deployed, sometimes regularly increasing the antidepressant dose during the winter months.

\section{Light therapy}

Efficacy

Some of the evidence for the efficacy of light therapy in winter depression can be criticised on the grounds that placebo-controlled trials have been conducted on knowledgeable, often self-diagnosed subjects who could not have been blind to treatment allocation and anticipated improvement with bright light. It is also difficult to envisage many interventions more likely to exert a placebo effect than spending time each day sitting in front of a bright light source. Given these considerations, several well-designed trials have found light therapy to be significantly beneficial for winter depression, and the best evidence of efficacy is that bright light in the mornings is more helpful than bright light in the evenings (Lewy et al, 1998). This also underlines the logic of the phase-shift hypothesis mentioned above. Thompson's (2001) meta-analysis concluded that treatment with a lightbox in seasonal affective disorder had a number needed to treat of between 4 and 5, which compares well with many medical interventions.

\section{Predictors of outcome}

Features associated with a good response to light therapy include hypersomnia, increased appetite, winter weight gain and complete remission of symptoms in summer. Factors that have been found, less predictably, to correlate with better outcomes include feeling worse in the morning (possibly a phase-shift phenomenon), young age and eating a lot of sweet foodstuffs late in the day.

\section{Timescales of response and treatment}

If light therapy is to prove successful, response often commences within days and is usually apparent by the end of week one. In general, if there has been no response in the first 3 weeks (given adequate adherence to treatment), then a subsequent response is unlikely. For this reason, it is appropriate that some lightbox suppliers have a sale or return policy within 3 weeks of initial purchase. If light therapy is effective, then usually it can be discontinued for a few days (e.g. during a weekend away from home) without too many deleterious effects.

It is usually advisable, especially if patients are buying their own lightbox, to advocate that light therapy commences for the first time only once symptoms are clearly present; only then can effectiveness be assessed. However, in future winters, patients who respond to light therapy should usually start treatment as soon as the first symptom emerges. Although it has not been evident 
in my own clinical practice, light therapy prior to the onset of symptoms might prevent a winter episode of depression (Partonen \& Lonnqvist, 1996). Light therapy is usually phased out gradually in spring, often round about the time the clocks change, but this will depend on previous timing of symptom offset and current well-being.

\section{Light and exercise}

The benefits of winter exercise outdoors have been mentioned already. Leppamaki et al (2002) described a possible adjunctive effect of exercise and bright morning light. For patients with winter depression, this would generally comprise exercising on a treadmill or stationary bicycle while using a lightbox or light visor.

\section{Side-effects of light therapy}

Headaches and blurred vision are the most commonly reported adverse effects, occurring in up to $20 \%$ of patients. Feeling 'wired' (anxiously energetic as if after too much caffeine) is also fairly common. Rarely, hypomania may be precipitated. If used too late in the day, especially in people who are responders to light therapy, insomnia may well be induced. Light therapy might cause problems for patients on potentially photosensitising medication such as chlorpromazine, lithium or St John's Wort. People with pre-existing retinitis pigmentosa or macular degeneration are advised to have an opthalmological examination before treatment, although there are no known cases of opthalmological damage attributable to light therapy. There is, however, one recent report (Swiecicki \& Szafranski, 2002) of possible serotonin syndrome when used in tandem with SSRIs.

\section{Light therapy in other phase-shifted conditions}

The use of light therapy in jetlag, sometimes in combination with melatonin, is quite well accepted. It has also been used effectively in delayed sleep phase disorder (common in adolescence), and timed bright light can enhance adaptation to shift work. In older people with dementia, dawn and/or dusk simulation (see below) can improve disturbed sleep/wake cycles.

\section{How should a lightbox be used?}

The main points are highlighted in Box 1. Brightness of light is measured in lux, and lux vary with the square of the distance from a light source (e.g. double the distance, one quarter of the brightness). For this reason, it is important for the eyes of subjects seeking an adequate 'dose' of light therapy to be sufficiently close to their lightbox. It is usual to aim for an
Box 1 Practical aspects of light therapy for winter depression

- It is most effective in the early morning.

- If it can be tolerated, users aim for a brightness of 10000 lux.

- 30 minutes each morning is an appropriate initial duration of treatment.

- Responders usually improve within one week and not later than 3 weeks.

- Side-effects include headaches, blurred vision, insomnia and overactivity.

- There are alternatives to lightboxes, which include light visors and dawn simulating alarm clocks.

illuminance as high as 10000 lux, which equates to the eyes being 18 to 21 inches away from an average lightbox, and to start by using it for 30 minutes each morning. Earlier recommendations, giving rise to a roughly equivalent dose, had tended to be for a lesser brightness of around 2500 lux for about 2 hours, but most people regarded this as too time-consuming. Response and side-effects are titrated against light dose. Thus, incomplete responders use their lightboxes for longer (sometimes requiring them at work as well as at home), and sensitive responders can use them for less than 30 minutes. Some people may benefit from another exposure later in the day, but in general lightboxes should not be used after $17.00 \mathrm{~h}$, because of the risk of insomnia. Other sideeffects can usually be managed successfully by retreating slightly from the lightbox or by using it for less time. Headaches and blurred vision can be lessened by gazing past the light source rather than staring at it fixedly.

\section{Which lightbox?}

In the UK, lightboxes currently retail at between $£ 100$ and $£ 250$, and the boxes around the middle of that price range generally afford a good balance between brightness, portability and cost. Smaller units can be fitted to the top of computer screens, and their portability allows them to be used away from home. Users have to position their eyes quite close to smaller units to achieve an adequate dose, if this constitutes their only lightbox. Two reputable lightbox suppliers are Outside In (Cambridge) and Lightbox (Glasgow). They both operate a 21-day sale or return policy. Advice about light therapy and other aspects of seasonal affective disorder can be accessed through the SADAssociation (PO Box 989, Steyning, BN44 3HG, UK. http://www.sada. org.uk). 


\section{Alternatives to lightboxes}

Desk lamps and small lightboxes are frequently used at work for those requiring longer or less bright exposure. Two other alternatives to lightboxes are light visors and dawn-simulating alarm clocks.

Rechargeable light visors are similar to baseball caps with small light sources in the brim shining into the wearer's eyes. Older versions were rather heavy and cumbersome, but more recent designs are more comfortable to wear. Visors can be useful when it is impractical to sit still in front of a lightbox, perhaps most commonly among mothers of young children. Although anecdotal accounts of the efficacy of light visors are very often positive, the research evidence to support their use is not compelling.

There is more evidence to support the effectiveness of dawn-simulating alarm clocks. These come on at dim illuminance and gradually increase in brightness over a period of 30 to 90 minutes leading up to the person's normal wakening time. A study by Avery et al (2001) found them to be more efficacious in patients with winter depression and hypersomnia than lightboxes or placebo (dim red) dawnsimulating alarm clocks. This may relate partly to the difficulties that depressed, oversleeping patients sometimes encounter in complying adequately with 30 minutes of morning lightbox therapy; dawnsimulating alarm clocks require less energy and commitment. In our own service, we have been lending dawn-simulating alarm clocks to patients with established seasonal affective disorder, usually as an adjunct to lightboxes, and they usually report wakening feeling more refreshed and alert. Some of these alarm clocks also simulate dusk, through a reciprocal dimming down function that can be useful for those who have difficulty falling asleep. Dawnsimulating alarm clocks are of much lower illuminance than lightboxes and, given their use while patients are asleep, a great deal less light enters the eyes. It is hypothesised that they exert their therapeutic effects because the light stimulation occurs at a particularly sensitive point on humans' light-phase response curve, as we are especially reactive to changes in light levels around dawn.

\section{References}

American Psychiatric Association (1994) Diagnostic Criteria from DSM-IV. Washington, DC: APA.

Avery, D. H., Elder, D. N., Bolte, M. A., et al (2001) Dawn simulation and bright light in the treatment of SAD: a controlled study. Biological Psychiatry, 50, 205-216.

Blouin, A. G., Blouin, J. H., Aubin, P., et al (1992) Seasonal pattern of bulimia nervosa. American Journal of Psychiatry, $149,73-81$.

Eagles, J. M., Wileman, S. M., Cameron, I. M., et al (1999) Seasonal affective disorder among primary care attenders and a community sample in Aberdeen. British Journal of Psychiatry, 175, 472-475.
Eagles, J. M., Howie, F. L., Cameron, I. M., et al (2002) Use of health care services in seasonal affective disorder. British Journal of Psychiatry, 180, 449-454.

Hesselmann, B., Habeler, A., Praschak-Reider, N., et al (1999) Mirtazapine in seasonal affective disorder (SAD): a preliminary report. Human Psychopharmacology, 14, 59-62.

Kasper, S., Rogers, S. L. B., Yancey, A., et al (1989) Phototherapy in individuals with and without subsyndromal seasonal affective disorder. Archives of General Psychiatry, 46, 837-844.

Leppamaki, S., Partonen, T. \& Lonnqvist, J. (2002) Brightlight exposure combined with physical exercise elevates mood. Journal of Affective Disorders, 72, 139-144.

Lewy, A. J., Sack, R. L., Miller, L. S., et al (1987) Antidepressant and circadian phase-shifting effect of light. Science, 253, 352-354.

Lewy, A. J., Bauer, B. K., Cutler, N. L., et al (1998) Morning vs evening light treatment of patients with winter depression. Archives of General Psychiatry, 55, 890-896.

Magnusson, A. (2000) An overview of epidemiological studies on seasonal affective disorder. Acta Psychiatrica Scandinavica, 101, 176-184

Magnusson, A. \& Stefansson, J. G. (1993) Prevalence of seasonal affective disorder in Iceland. Archives of General Psychiatry, 50, 941-946.

Mersch, P. P. A., Middendorp, H. M., Bouhuys, A. L., et al (1999) Seasonal affective disorder and latitude: a review of the literature. Journal of Affective Disorders, 53, 35-48.

Michalak, E. E., Wilkinson, C., Dowrick, G., et al (2001) Seasonal affective disorder: prevalence, detection and current treatment in North Wales. British Journal of Psychiatry, 179, 31-34.

Michalak, E. E., Wilkinson, C., Hood, K., et al (2003) Seasonality, negative life events and social support in a community sample. British Journal of Psychiatry, 182, 434438 .

Neumeister, A., Turner, E. H. Matthews, J. R., et al (1998) Effects of tryptophan depletion vs catecholamine depletion in patients with seasonal affective disorder in remission with light therapy. Archives of General Psychiatry, 55, 524530.

Partonen, T. \& Lonnqvist, J. (1996) Prevention of winter seasonal affective disorder by bright-light treatment. Psychological Medicine, 26, 1075-1080.

Partonen, T. \& Magnusson, A. (eds) (2001) Seasonal Affective Disorder: Practice and Research. Oxford: Oxford University Press.

Reichborn-Kjennerud, T., Lingjaerde, O. \& Dahl, A. A. (1997) DSM-III personality disorders in seasonal affective disorders: change associated with depression. Comprehensive Psychiatry, 38, 43-48.

Rohan, K. J., Sigmon, S. T. \& Dorhofer, D. M. (2003) Cognitive-behavioral factors in seasonal affective disorder. Journal of Consulting and Clinical Psychology, 71, 22-30.

Rosenthal, N. E., Sack, D. A., Gillin, J. C., et al (1984) Seasonal affective disorder: a description of the syndrome and preliminary findings with light therapy. Archives of General Psychiatry, 41, 72-80.

Rosenthal, N. E., Genhart, M., Sack, D. A., et al (1987) Seasonal affective disorder: relevance for treatment and research of bulimia. In Psychology of Bulimia (eds J. I. Hudson \& H. G. Pope), pp. 205-208. Washington, DC: APA.

Swedo, S. E., Pleeter, J. D., Richter, D. M., et al (1995) Rates of seasonal affective disorder in children and adolescents. American Journal of Psychiatry, 152, 1016-1019.

Swiecicki, L. \& Szafranski, T. (2002) Side effects after phototherapy implementation in addition to fluoxetine or sertraline treatment: a report of two cases. World Journal of Biological Psychiatry, 3, 109-111.

Thompson, C. (2001) Evidence-based treatment. In Seasonal Affective Disorder: Practice and Research (eds T. Partonen \& A. Magnusson), pp. 151-158. Oxford: Oxford University Press.

Thompson, C. \& Isaacs, G. (1988) Seasonal affective disorder in a British sample: symptomatology in relation to mode of referral and diagnostic sub-type. Journal of Affective Disorder, 14, 1-11. 


\section{Multiple choice questions}

1 The following are typical of winter depression:

a high rates of suicide in December

b weight loss

c early morning wakening

d daytime somnolence

e carbohydrate avoidance.

2 Sufferers of winter depression:

a are most commonly women during their reproductive years

b typically remit completely in summer

c usually benefit by moving away from the equator

d are never symptomatic before puberty

e frequently present with somatic symptoms.

3 Typical patients with winter depression benefit from:

a benzodiazepines

b bright light at $23.00 \mathrm{~h}$. c sunny winter holidays

d electroconvulsive therapy

e sertraline.

4 Light therapy for winter depression:

a is more effective in the morning than in the evening

b should be administered while the patient's eyes are closed

c is optimally used once weekly

$\mathrm{d}$ helps to improve insomnia

e corrects circadian phase delay.

5 Recognised uses of light therapy include:

a treatment of jetlag

$b$ treatment of headache

c treatment of delayed sleep phase disorder

d treatment of sleep/wake cycle disorders in people with dementia

e adaptation to shift working.

\section{MCQs in Psychiatry}

By David McNamara

Practising multiple choice questions is a superb way to reinforce knowledge.

This book contains some 600 questions covering all the sub-specialties of psychiatry. It is intended for use in conjunction with the College Seminars series and will be of great benefit to all trainees and established clinicians. The book will enable users to identify sgaps in their learning and to consolidate their knowledge and understanding of psychiatry.

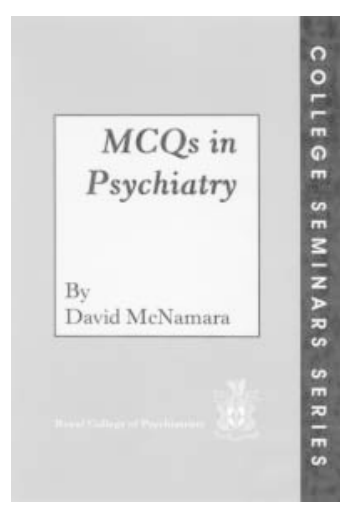

2003, 256pp, Paperback, ISBN 1901242 89 7, Price $£ 18.00$

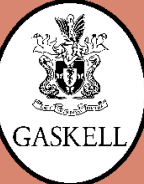

